\title{
Optical Manipulation with Plasmonic Beam Shaping Antenna Structures
}

\author{
Young Chul Jun ${ }^{1,2}$ and Igal Brener1,2 \\ ${ }^{1}$ Center for Integrated Nanotechnologies (CINT), Sandia National Laboratories, Albuquerque, NM 87185, USA \\ ${ }^{2}$ Sandia National Laboratories, Albuquerque, NM 87185, USA
}

Correspondence should be addressed to Young Chul Jun, youngchul.jun@sandia.gov

Received 6 June 2012; Accepted 16 July 2012

Academic Editor: Alexandra E. Boltasseva

Copyright ( $) 2012$ Y. C. Jun and I. Brener. This is an open access article distributed under the Creative Commons Attribution License, which permits unrestricted use, distribution, and reproduction in any medium, provided the original work is properly cited.

Near-field optical trapping of objects using plasmonic antenna structures has recently attracted great attention. However, metal nanostructures also provide a compact platform for general wavefront engineering of intermediate and far-field beams. Here, we analyze optical forces generated by plasmonic beam shaping antenna structures and show that they can be used for general optical manipulation such as guiding of a dielectric particle along a linear or curved trajectory. This removes the need for bulky diffractive optical components and facilitates the integration of optical force manipulation into a highly functional, compact system.

\section{Introduction}

Optical forces can be exerted on objects by transferring light momentum. A tightly focused optical field can produce a stable trapping force around its focus and is highly useful for trapping particles or biological molecules; this effect is known as "optical tweezers" [1-3]. More generally, carefully tailored wavefronts of light can be utilized for various types of optical manipulation such as particle guiding and rotation [4-6]. Recently, plasmonic nanostructures have attracted attention for this application [7-9]. A variety of nanopatterns such as metallic nanoparticles [10] and sharp tips [11] have been considered for plasmonic optical tweezers. Those structures have tighter light confinement and greater field gradient than conventional optical tweezers and thus produce a larger trapping force and smaller trapping volume.

However, plasmonic antenna structures are also useful for shaping intermediate and far-field beam patterns. Metal nanostructures provide a general platform for wavefront engineering $[12,13]$. Metal slitgroove or hole-groove structures were shown to be useful for collimating/directing light [14-16]. Metal nanoslit or nanohole arrays were used for focusing a beam into a small spot [17-19]. Metal nanostructures were also studied for angular momentum control of light [20-22]. Previously, such beam shaping has been achieved with elaborate wavefront and beam control (such as Bessel, Airy, or Laguerre-Gaussian beams) involving bulky diffractive or holographic components [5]. Nanostructured metal films provide an alternative, compact way for tailoring wavefronts without such bulky and complicated elements.

In this paper, we analyze optical forces caused by nanostructured metal films and show that they can be used for general optical manipulation. The force magnitude is comparable to that achieved with conventional diffractive optics, but the plasmonic nanostructures have larger design and patterning flexibility. Moreover, the planar geometry facilitates integration with other components, such as lasers [23] and optical fibers [24]. They can be integrated with microfluidics or micromechanical systems too. Thus, we expect that plasmonic beam shaping will give another interesting venue for optical manipulation studies, especially promising for compact integrated systems.

\section{Guiding along a Linear Trajectory}

We first consider optical forces using symmetric metal slitgroove structures. Lezec et al. demonstrated narrow angle $\left( \pm 3^{\circ}\right)$ light collimation using such structures [14]. Light still diverges, but it can be collimated tightly over a nonnegligible 
distance (more than tens of micrometers for visible light). Figure 1(a) shows a plasmonic beaming structure exhibiting such light collimation in water. A metal slit is surrounded by 6 side grooves on each side. The optimal groove parameters for beaming were chosen using numerical simulations (Figure 1 (a) caption). Laser light $\left(\lambda_{0}=633 \mathrm{~nm}\right)$ is incident from the left and collimated after passing the slitgroove.

An incident plane wave excites surface plasmon waves in the slit, and those surface plasmons are scattered into far-field light again at the exit slit. The properly designed sidegrooves produce the constructive interference of scattered light and a collimated, narrow-angle beam as shown in Figure 1(b) [25]. The incident side of the metal film can be also patterned to increase the optical power throughput by orders of magnitude [26]. This will reduce the required incident laser power for optical manipulation. However, it is well known that the beaming pattern is determined solely by the groove pattern on the output side.

We now calculate the optical forces and show that this beaming structure can be used for dielectric particle guiding. A dielectric sphere (diameter $D=1 \mu \mathrm{m}, n=1.59$ ) is placed around the collimated beam, and we perform numerical simulations (3-dimensional finite difference time domain, FDTD) [27] to obtain the total electromagnetic fields (including both incident and scattered fields). Then, we evaluate the optical force on a particle by calculating and integrating Maxwell's stress tensor over a surface surrounding the particle. The time-averaged optical force is

$$
\langle\mathbf{F}\rangle=\int\langle\mathbf{T}\rangle \cdot \mathbf{n} d a
$$

where $\mathbf{n}$ is the unit vector normal to the surface, and $\mathbf{T}$ is the Maxwell's stress tensor [28] given by

$$
\mathbf{T}=\varepsilon \varepsilon_{0} \mathbf{E E}+\mu \mu_{0} \mathbf{H} \mathbf{H}-\frac{\mathbf{I}}{2}\left(\varepsilon \varepsilon_{0}|\mathbf{E}|^{2}+\mu \mu_{0}|\mathbf{H}|^{2}\right) .
$$

Figure 1(c) shows optical forces for a particle located $12 \mu \mathrm{m}$ away from the film. The force in the $Y$-direction $\left(F_{Y}\right)$ has a negative slope around the beam center (which means the particle is attracted toward the beam center), and the force in the $X$-direction $\left(F_{X}\right)$ is positive (which means the particle is pushed forward). We calculate the pushing force $F_{X}$ as a function of the particle diameter and find it increasing rapidly with size in the considered range (Figure $1(\mathrm{~d})$ ). We gradually move a particle (within the dotted region in Figure 1(b)) and obtain the force vector map (Figure 1(e)). The arrows indicate the direction and magnitude of optical forces at a corresponding position. A particle is trapped into the center of the collimated beam due to the gradient force and is pushed forward along a linear trajectory due to the scattering force.

Such optical guiding has been achieved with nondiffractive or propagation-invariant laser modes (such as Bessel beams) which are generated by diffractive or holographic optical elements $[5,29]$. The origin of nondiffracting beams can be explained by the decomposition of a field into plane waves. Each plane wave component acquires an equal phase shift as the field propagates in such beams. This makes the sum of plane waves invariant, generating diffraction-free behavior over a limited distance range. The nanopatterned metal films provide a simple, alternative way for particle guiding over a nonnegligible distance.

\section{Guiding along a Tilted or Curved Trajectory}

We can also guide a particle at a predefined direction with a slightly modified structure. An asymmetric slitgroove structure shown in Figure 2(a) generates a collimated beam at an angle (Figure 2(b)). The different groove periods on either side generate constructive interference in the off-axis direction. As in the previous section, we optimize the asymmetric groove parameters through numerical simulations. The calculated force vector map for this structure (Figure 2(c)) shows that a particle is trapped and guided into the off-axis direction; the trajectory is also curved. The slitgroove structures in Figures 1 and 2 extend in the $Z$-direction. Thus, the collimated beam also extends in the $Z$-direction and this is good for manipulating elongated particles or molecules. Moreover, we could also use a holegroove or bull's eye structure [14] to get 2-dimensional collimation. Then, a particle will be trapped and guided along an axial beam. With shifted hole/groove positions, the axial beam can be also directed off-axis [30], similar to Figure 2(b).

Such curved optical guiding can also be achieved using sophisticated optical beam modes. For example, Airy beams were recently used to guide a dielectric particle along a curved trajectory over $75 \mu \mathrm{m}$, termed "optical snowblowing" [31]. Airy beams do not propagate along straight lines but instead follow parabolic trajectories. Such beams can be used to transfer particles and cells between microfluidic chambers or remove particles from a region of space. Plasmonic antenna structures provide a simple, alternative way for such optical manipulation.

Similar guiding of a particle has been demonstrated using waveguide structures too [32-35]. A dielectric particle can be trapped by the evanescent field of waveguide modes and pushed along the waveguide toward the light propagation direction. In contrary to this waveguide method, the plasmonic beam shaping enables such guiding even in regions beyond the extent of evanescent fields (intermediate to far field). Furthermore, plasmonic beam shaping has larger design flexibility and tunability.

It is possible to obtain dynamic tuning in our beaming and optical manipulation system. Figure 3 shows the simulated field intensities for two incident light wavelengths $\left(\lambda_{0}=\right.$ $560 \mathrm{~nm}$ and $700 \mathrm{~nm}$ ). The pattern geometry is the same as Figure 2(a) (i.e., asymmetric slitgroove). We notice that the shorter wavelength light $\left(\lambda_{0}=560 \mathrm{~nm}\right)$ is directed along the center line (Figure 3(a)), while the long wavelength light $\left(\lambda_{0}\right.$ $=700 \mathrm{~nm})$ is directed upward $(Y>0$, Figure 3(b)). Different wavelengths result in different interference conditions and beaming directions. This also implies that a particle will be pushed into different directions depending on the incident laser wavelength. If we use two different wavelength laser sources or a tunable laser, we can change the optical force directions. Moreover, light diffraction in these slitgroove 


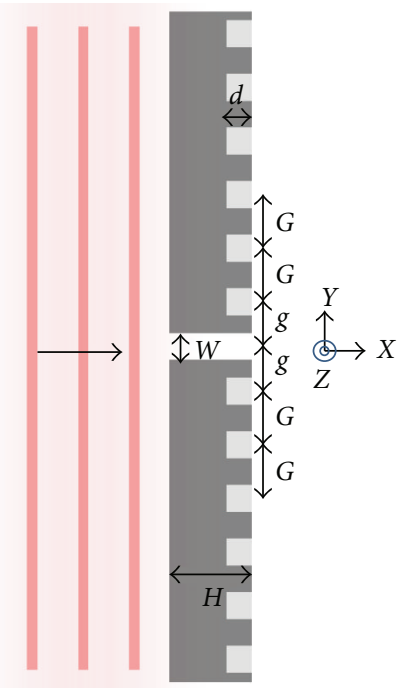

(a)

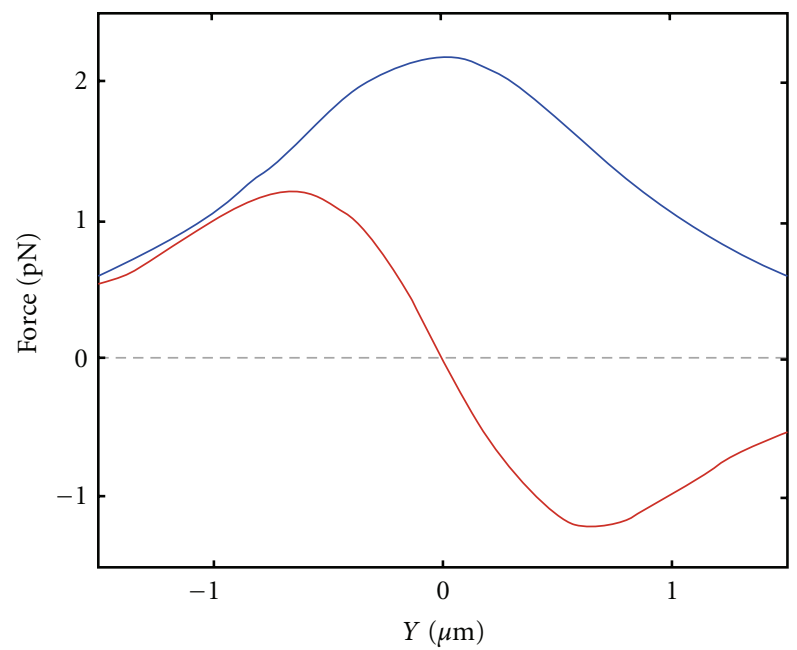

$-F_{X}$

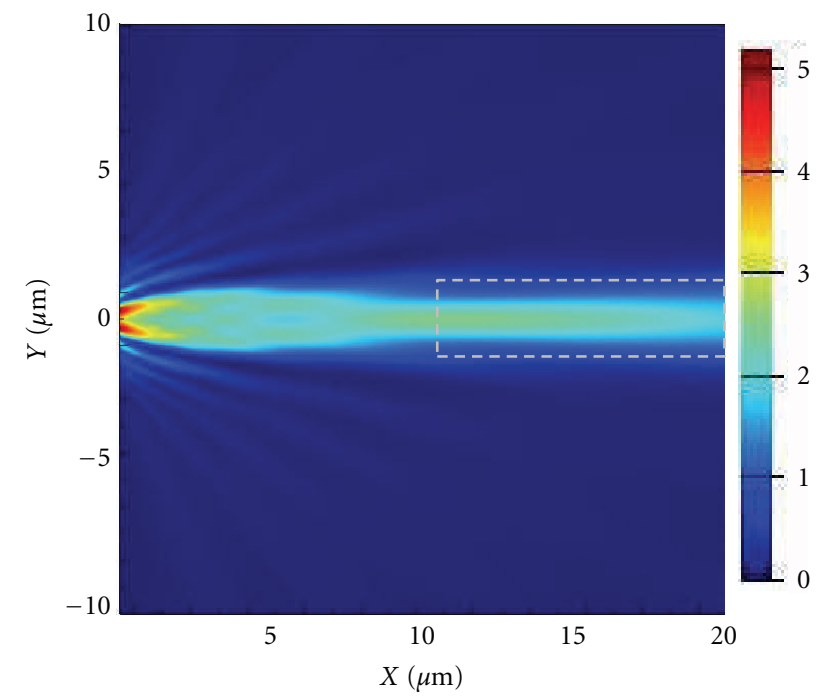

(b)

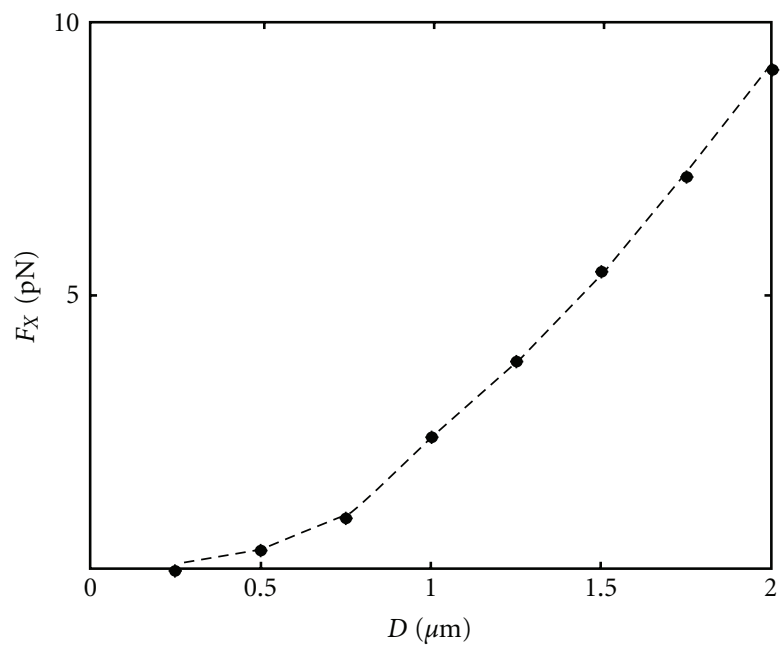

(c)

(d)

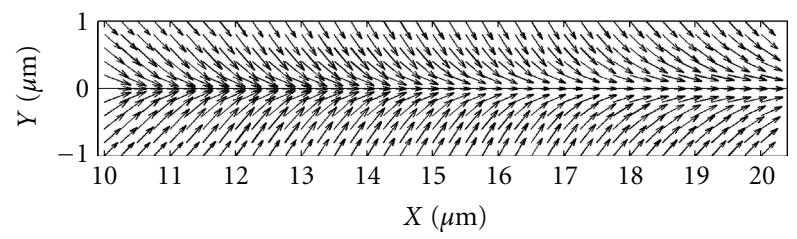

(e)

Figure 1: (Color online) Optical guiding with a symmetric slitgroove along a linear trajectory. (a) Schematic of the silver slitgroove. $W=$ $80 \mathrm{~nm}, G=g=415 \mathrm{~nm}, d=40 \mathrm{~nm}$, and $H=300 \mathrm{~nm}$. The film is immersed in water $(n=1.33)$. The structure is extended in the $Z$-direction. The optimal geometry for beaming was determined by numerical simulations. (b) Electric field intensity obtained from a numerical simulation. (c) Optical force for a dielectric particle $(D=1 \mu \mathrm{m}, n=1.59)$ located at $X=12 \mu \mathrm{m}$. The force magnitude is normalized to the transmitted power (through the slit) of $10 \mathrm{~mW} / \mu \mathrm{m}$ in the $Z$-direction. (d) Optical force $F_{X}$ as a function of the particle diameter. The particle is located at $X=12 \mu \mathrm{m}, Y=0 \mu \mathrm{m}$. (e) Optical force vector map. 


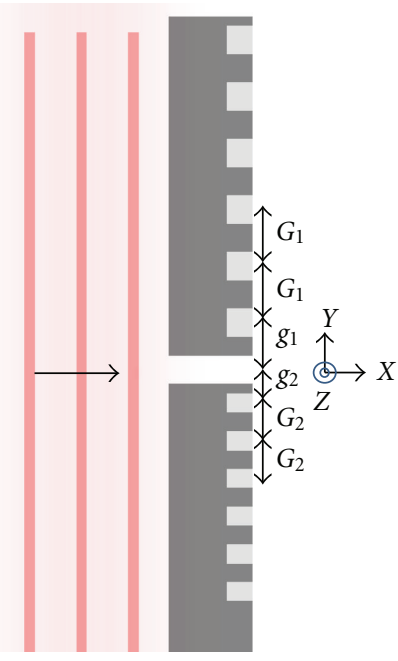

(a)

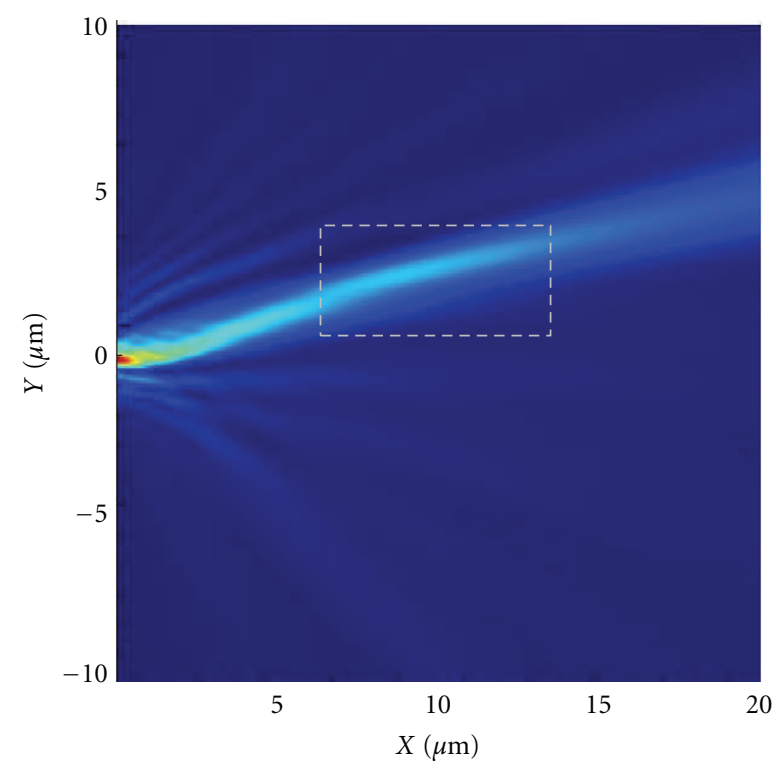

(b)

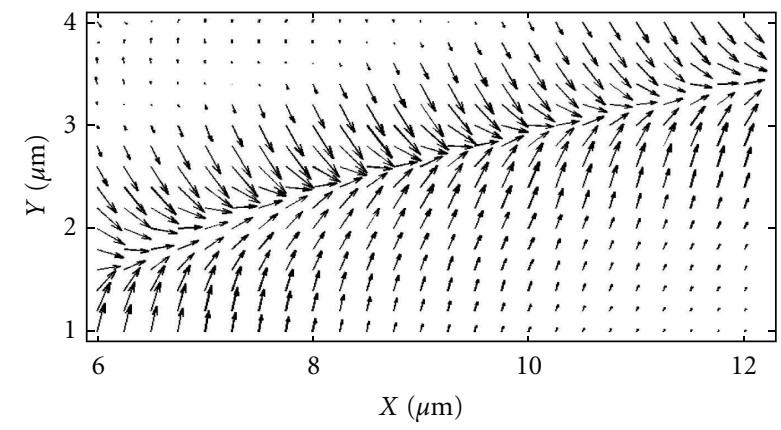

(c)

Figure 2: (Color online) Optical guiding with an asymmetric slitgroove. (a) Schematic of the silver slitgroove. $G_{1}=g_{1}=500 \mathrm{~nm}, G_{2}=$ $g_{2}=320 \mathrm{~nm}, W=80 \mathrm{~nm}, d=40 \mathrm{~nm}$, and $H=300 \mathrm{~nm}$. (immersed in water). (b) Electric field intensity showing the plasmonic beaming at off-axis. (c) Optical force vector map for the dotted region in (b).

systems is sensitive to the refractive index of a medium inside the slit or on the grooves. There have been studies for dynamic control of metal slit transmission using nonlinear optical medium [36] or optical interference with a control beam [37]. Thus, if we dynamically tune the refractive index either optically or electrically, we can actively control optical force directions without using any moving parts.

\section{4. “On-Chip” Optical Rotation}

Light also possesses angular momentum, and the transfer of angular momentum can lead to rotational motion of a particle (by exerting optical torques on the object). For a monochromatic field, the time-averaged optical torque [8] can be obtained as

$$
\langle\mathbf{N}\rangle=-\int\langle\mathbf{T}(\mathbf{r}, t) \times \mathbf{r}\rangle \cdot \mathbf{n}(\mathbf{r}) d a,
$$

where $\mathbf{n}$ is the unit vector normal to a surface surrounding the particle, and $\mathbf{T}$ is the Maxwell's stress tensor.
Nanopatterned metal films have also been used for angular momentum control of light. Linearly polarized light can be converted into circularly polarized one (that possesses the spin angular momentum of $h / 2 \pi$ per photon), for example, by passing through an elliptical metal hole-groove structure (Figure 4(a)) [20] or coupled orthogonal metal slits [21]. These structures effectively work as a plasmonic quarter waveplate. Recently, a plasmonic spiral waveplate was demonstrated, consisting of arrays of rotated $\mathrm{V}$-shaped plasmonic antennas $[22,38]$. This structure generates a helical wavefront that possesses the orbital angular momentum of $l h / 2 \pi$ per photon (where $l$ is an integer). V-shaped plasmonic antennas impart abrupt phase shifts to propagating light. By arranging continuously rotated V-shaped antennas (Figure 4(b)) on a transparent substrate, we can implement a plasmonic spiral waveplate. Laguerre-Gaussian laser modes are typically used to generate such a helical wavefront [3]. But metal nanostructures provide an alternative, compact way to produce it without bulky diffractive optical components. We can potentially combine these plasmonic structures with a 


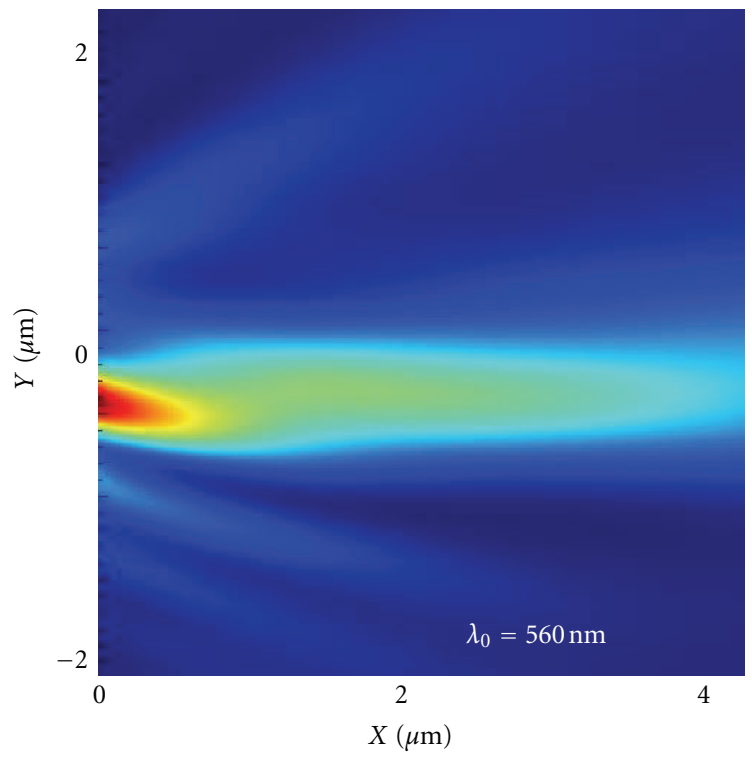

(a)

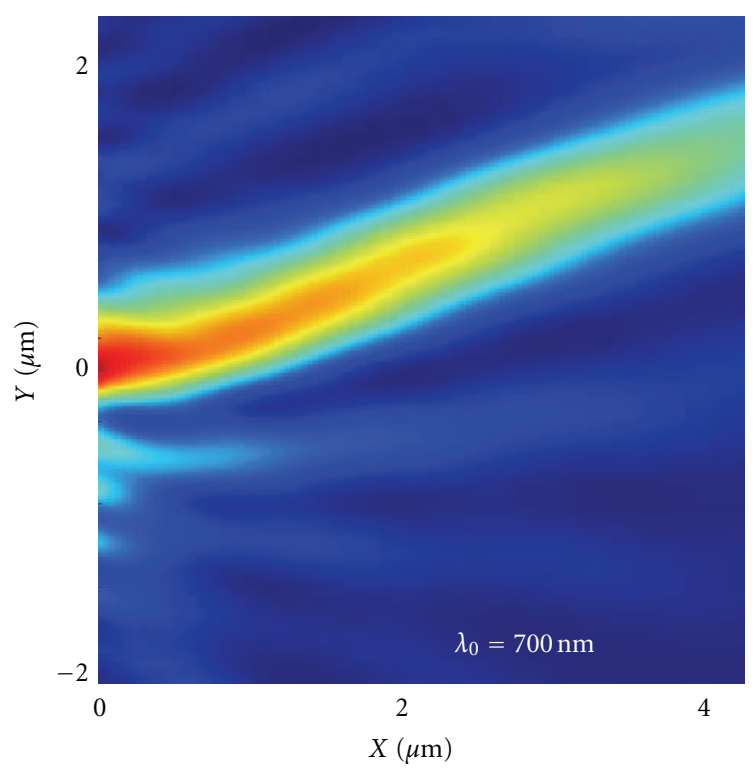

(b)

FIGURE 3: (Color online) Wavelength-dependent beam steering from an asymmetric slitgroove. Electric field intensities of the transmitted light are shown at two different wavelengths.

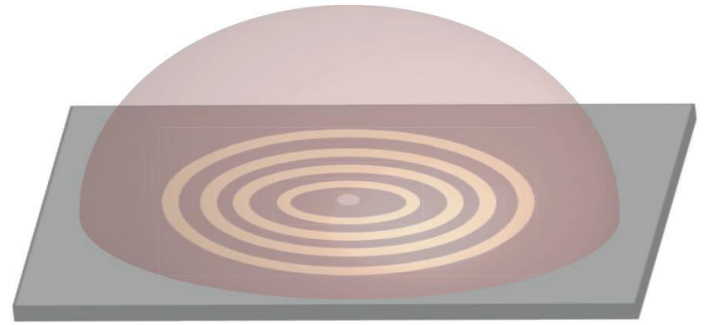

(a)

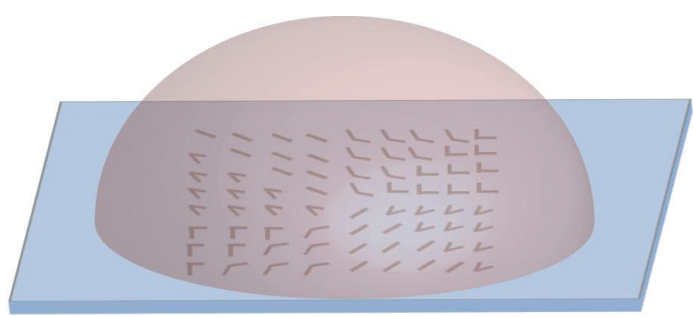

(b)

FIGURE 4: (Color online) Schematics for the "on-chip" optical rotation. A dielectric microlens is integrated with (a) a plasmonic quarter waveplate (milled on a metal film) or (b) a plasmonic spiral waveplate (consisting of V-antenna arrays on a transparent substrate). Linearly polarized light is incident from below and focused on top passing through the microlens. Objects can be trapped at the focus and be rotated on a chip.

dielectric microlens to achieve optical trapping and rotation simultaneously (Figure 4). This will be an interesting topic of future investigation.

\section{Conclusion}

In summary, we have studied optical forces generated by plasmonic beam shaping antenna structures and find that they are suitable for general optical manipulation, such as guiding of a dielectric particle along a linear or curved trajectory. The planar geometry and design flexibility make them ideal for large array fabrication. This could enable a multitude of new opportunities for integrating optical manipulation into compact, chip-scale systems.

\section{Acknowledgments}

This work was performed, in part, at the Center for Integrated Nanotechnologies, a U.S. Department of Energy,
Office of Basic Energy Sciences user facility. Sandia National Laboratories is a multiprogram laboratory managed and operated by Sandia Corporation, a wholly owned subsidiary of Lockheed Martin Corporation, for the U.S. Department of Energy's National Nuclear Security Administration under Contract DE-AC04-94AL85000.

\section{References}

[1] A. Ashkin, J. M. Dziedzic, J. E. Bjorkholm, and S. Chu, "Observation of a single-beam gradient force optical trap for dielectric particles," Optics Letters, vol. 11, no. 5, pp. 288-290, 1986.

[2] A. Ashkin, "History of optical trapping and manipulation of small-neutral particle, atoms, and molecules," IEEE Journal on Selected Topics in Quantum Electronics, vol. 6, no. 6, pp. 841856,2000

[3] K. C. Neuman and S. M. Block, “Optical trapping," Review of Scientific Instruments, vol. 75, no. 9, pp. 2787-2809, 2004.

[4] D. G. Grier, "A revolution in optical manipulation," Nature, vol. 424, no. 6950, pp. 810-816, 2003. 
[5] K. Dholakia and T. Čižmár, "Shaping the future of manipulation," Nature Photonics, vol. 5, no. 6, pp. 335-342, 2011.

[6] M. Padgett and R. Bowman, "Tweezers with a twist," Nature Photonics, vol. 5, no. 6, pp. 343-348, 2011.

[7] M. L. Juan, M. Righini, and R. Quidant, "Plasmon nanooptical tweezers," Nature Photonics, vol. 5, no. 6, pp. 349-356, 2011.

[8] L. Novotny and B. Hecht, Principles of Nano-Optics, chapter 13, Cambridge University Press, 2006.

[9] X. Yang, Y. Liu, R. F. Oulton, X. Yin, and X. Zhang, "Optical forces in hybrid plasmonic waveguides," Nano Letters, vol. 11, no. 2, pp. 321-328, 2011.

[10] R. Quidant, D. Petrov, and G. Badenes, "Radiation forces on a Rayleigh dielectric sphere in a patterned optical near field," Optics Letters, vol. 30, no. 9, pp. 1009-1011, 2005.

[11] L. Novotny, R. X. Bian, and X. Sunney Xie, "Theory of Nanometric optical tweezers," Physical Review Letters, vol. 79, no. 4, pp. 645-648, 1997.

[12] L. Verslegers, P. B. Catrysse, Z. Yu, W. Shin, Z. Ruan, and S. Fan, "Phase front design with metallic pillar arrays," Optics Letters, vol. 35, no. 6, pp. 844-846, 2010.

[13] N. Yu and F. Capasso, "Wavefront engineering for midinfrared and terahertz quantum cascade lasers," Journal of the Optical Society of America B, vol. 27, no. 11, pp. B18-B35, 2010.

[14] H. J. Lezec, A. Degiron, E. Devaux et al., "Beaming light from a subwavelength aperture," Science, vol. 297, no. 5582, pp. 820 822, 2002.

[15] S. Kim, H. Kim, Y. Lim, and B. Lee, "Off-axis directional beaming of optical field diffracted by a single subwavelength metal slit with asymmetric dielectric surface gratings," Applied Physics Letters, vol. 90, no. 5, Article ID 051113, 4 pages, 2007.

[16] Y. C. Jun, K. C. Y. Huang, and M. L. Brongersma, "Plasmonic beaming and active control over fluorescent emission," Nature Communications, vol. 2, no. 1, Article ID 283, 2011.

[17] Z. Sun and H. K. Kim, "Refractive transmission of light and beam shaping with metallic nano-optic lenses," Applied Physics Letters, vol. 85, no. 4, pp. 642-644, 2004.

[18] L. Verslegers, P. B. Catrysse, Z. Yu et al., "Planar lenses based on nanoscale slit arrays in a metallic film," Nano Letters, vol. 9, no. 1, pp. 235-238, 2009.

[19] H. Gao, J. K. Hyun, M. H. Lee, J. C. Yang, L. J. Lauhon, and T. W. Odom, "Broadband plasmonic microlenses based on patches of nanoholes," Nano Letters, vol. 10, no. 10, pp. 41114116, 2010.

[20] A. Drezet, C. Genet, and T. W. Ebbesen, "Miniature plasmonic wave plates," Physical Review Letters, vol. 101, no. 4, Article ID 043902, 4 pages, 2008.

[21] E. H. Khoo, E. P. Li, and K. B. Crozier, "Plasmonic wave plate based on subwavelength nanoslits," Optics Letters, vol. 36, no. 13, pp. 2498-2500, 2011.

[22] N. Yu, P. Genevet, M. A. Kats et al., "Light propagation with phase discontinuities: generalized laws of reflection and refraction," Science, vol. 334, no. 6054, pp. 333-337, 2011.

[23] N. Yu, J. Fan, Q. J. Wang et al., "Small-divergence semiconductor lasers by plasmonic collimation," Nature Photonics, vol. 2, no. 9, pp. 564-570, 2008.

[24] S. Kang, H. E. Joe, J. Kim, Y. Jeong, B. K. Min, and K. Oh, "Subwavelength plasmonic lens patterned on a composite optical fiber facet for quasi-one-dimensional Bessel beam generation," Applied Physics Letters, vol. 98, no. 24, Article ID 241103, 3 pages, 2011.

[25] L. B. Yu, D. Z. Lin, Y. C. Chen et al., "Physical origin of directional beaming emitted from a subwavelength slit,"
Physical Review B, vol. 71, no. 4, Article ID 041405, 4 pages, 2005.

[26] F. J. García-Vidal, H. J. Lezec, T. W. Ebbesen, and L. MartínMoreno, "Multiple paths to enhance optical transmission through a single subwavelength slit," Physical Review Letters, vol. 90, no. 21, Article ID 213901, 4 pages, 2003.

[27] Lumerical solutions, http://www.lumerical.com/.

[28] J. D. Jackson, Classical Electrodynamics, Wiley, 3rd edition, 1998.

[29] V. Garcés-Chávez, D. McGloin, H. Melville, W. Sibbett, and K. Dholakia, "Simultaneous micromanipulation in multiple planes using a self-reconstructing light beam," Nature, vol. 419, no. 6903, pp. 145-147, 2002.

[30] J. P. Tetienne, R. Blanchard, N. Yu et al., "Dipolar modeling and experimental demonstration of multi-beam plasmonic collimators," New Journal of Physics, vol. 13, Article ID 053057, 2011.

[31] J. Baumgartl, M. Mazilu, and K. Dholakia, "Optically mediated particle clearing using Airy wavepackets," Nature Photonics, vol. 2, no. 11, pp. 675-678, 2008.

[32] S. Kawata and T. Sugiura, "Movement of micrometer-sized particles in the evanescent field of a laser beam," Optics Letters, vol. 17, no. 11, pp. 772-774, 1992.

[33] S. Kawata and T. Tani, "Optically driven Mie particles in an evanescent field along a channeled waveguide," Optics Letters, vol. 21, no. 21, pp. 1768-1770, 1996.

[34] A. H. J. Yang, S. D. Moore, B. S. Schmidt, M. Klug, M. Lipson, and D. Erickson, "Optical manipulation of nanoparticles and biomolecules in sub-wavelength slot waveguides," Nature, vol. 457, no. 7225, pp. 71-75, 2009.

[35] D. Erickson, X. Serey, Y. F. Chen, and S. Mandal, "Nanomanipulation using near field photonics," Lab on a Chip, vol. 11, no. 6, pp. 995-1009, 2011.

[36] C. Min, P. Wang, X. Jiao, Y. Deng, and H. Ming, "Beam manipulating by metallic nano-optic lens containing nonlinear media," Optics Express, vol. 15, no. 15, pp. 9541-9546, 2007.

[37] A. E. Çetin, K. Güven, and Ö. E. Müstecaplığlu, "Active control of focal length and beam deflection in a metallic nanoslit array lens with multiple sources," Optics Letters, vol. 35, no. 12, pp. 1980-1982, 2010.

[38] X. Ni, N. K. Emani, A. V. Kildishev, A. Boltasseva, and V. M. Shalaev, "Broadband light bending with plasmonic nanoantennas," Science, vol. 335, no. 6067, article 427, 427. 

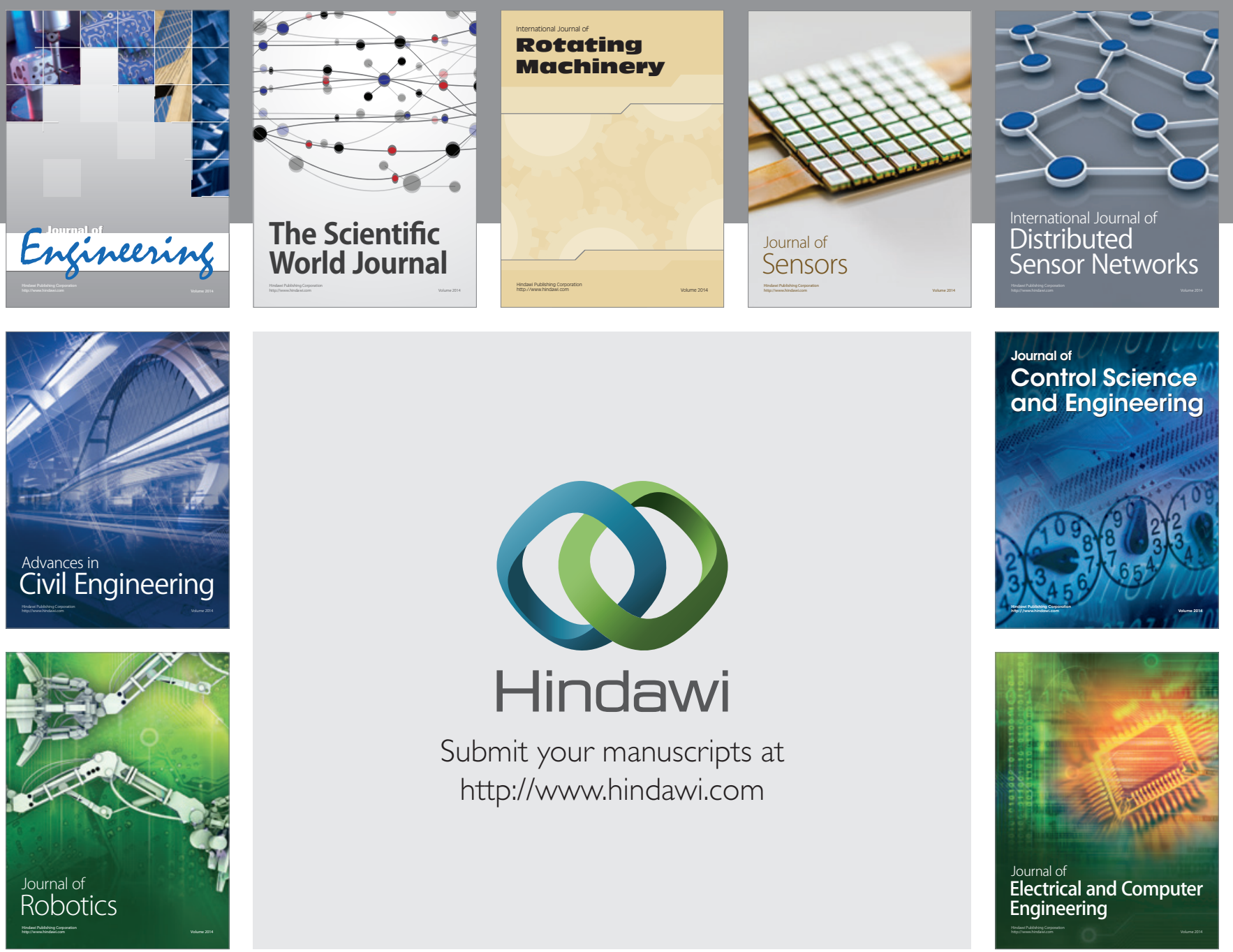

Submit your manuscripts at

http://www.hindawi.com
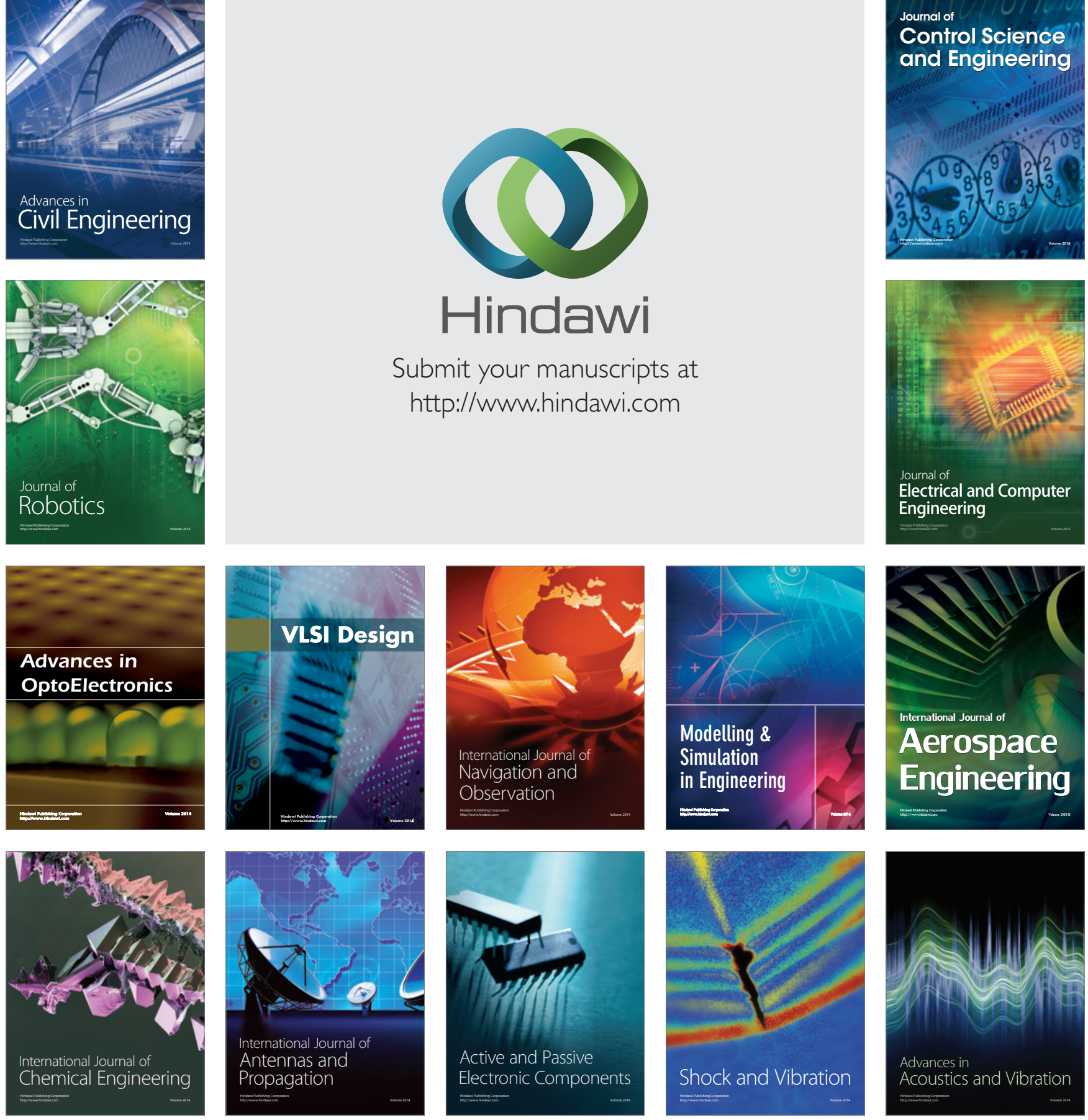